\title{
CURVATURE FORMS AND INTERACTION OF FIELDS
}

\author{
STOIL DONEV AND MARIA TASHKOVA
}

Communicated by Ivaïlo M. Mladenov

\begin{abstract}
We work out the general idea that a composite continuous physical system can be mathematically modelled locally as a completely integrable geometric distribution on a manifold, the time-recognizable subsystems to be modelled by corresponding subdistributions, and any local interaction between two subsystems of the physical system to be described in terms of the nonintegrability of the two subdistributions making use of the corresponding two curvature forms. As an illustration we present the corresponding description of photon-like objects, based on the notion that photon-like objects are real, massless time-stable physical objects with intrinsically compatible translational-rotational dynamical structure. The spatial propagation of the system follows some external/shuffling symmetry of the distribution.
\end{abstract}

\section{Introduction}

It hardly deserves to put under dispute the thesis that the concept of interaction in physics is a basic one. One of the most important in our view lessons that we more or less have been taught is that any detection and further study of a physical object requires some energy-momentum exchange. So, every physical object necessarily carries energy-momentum, every quantity of energy-momentum needs a carrier, and every interaction between two physical objects has an energy-momentum exchange aspect. The second lesson concerning any interaction is that, beyond its universality, energy-momentum is a conserved quantity, so NO loss of it is allowed: it may only pass from one object to another. This means, for example, also, that every annihilation process causes creation process(es), and the full energymomentum that has been carried by the annihilated objects, is carried away by the created ones. The energy-momentum exchange abilities of any physical object realize its protection against dangerous external influence on one side, and reveal its intrinsic nature, on the other side. Therefore, our knowledge about the entire complex of properties of a physical object relies on getting information about its abilities in this respect. 
The above views make us think and assume the standpoint that the most reliable dynamical equations, describing locally the time-evolution of a composite physical system, should express energy-momentum balance between/among its timerecognizable subsystems. So, finding natural appropriate mathematical structures in terms of which to understand and describe formally these exchange processes is of basic importance.

This paper aims to analyze the geometric structures and concepts connected with the problem of integrability of distributions on manifolds as possible useful and adequate tools in trying to peep into the intrinsic dynamical structure of photon-like objects. The basic view that we follow is that these objects consist of two timerecognizable and energy-momentum exchanging field subsystems, the mathematical image of each can be represented by a two dimensional nonintegrable subdistribution of a three dimensional integrable distribution on Minkowski space-time, and the corresponding two curvature forms represent formally the local dynamical equilibrium between the two subsystems.

\section{Basic Geometry}

\subsection{Distributions and Co-distributions}

We recall some facts from the geometry of distributions on manifolds [1,2]. Let $M$ be an arbitrary $n$-dimensional manifold. At every point $x \in M$ the tangent space $T_{x}(M)$ is defined. The union of all these spaces with respect to the points of $M$ defines the tangent bundle. On the other hand, the union of the co-tangent spaces $T_{x}^{*}(M)$ defines the co-tangent bundle. At every point now of $M$ we separate a $p$ dimensional subspace $\Delta_{x}(M) \subset T_{x}(M)$ in a smooth way, i.e., the map $x \rightarrow \Delta_{x}$ ix smooth. If this is done we say that a $p$-dimensional distribution $\Delta$ on $M$ is defined. From the elementary linear algebra we know that every $p$-dimensional subspace $\Delta_{x} \subset T_{x}(M)$ defines unique $(n-p)$-dimensional subspace $\Delta_{x}^{*} \subset T_{x}^{*}(M)$, such that all elements of $\Delta_{x}^{*}$ annihilate (i.e., send to zero) all elements of $\Delta_{x}$. In this way we get a $(n-p)$-dimensional co-distribution $\Delta^{*}$ on $M$.

We consider those vector fields, the representatives of which at every point are elements of the distribution $\Delta$, and those one-forms, the representatives of which at every point are elements of the co-distribution $\Delta^{*}$. Clearly, every system of $p$ independent and non-vanishing vector fields, belonging to $\Delta$, defines $\Delta$ equally well, and in this case we call such a system a differential $p$-system $\mathcal{P}$ on $M$. The corresponding system $\mathcal{P}^{*}$ of $q$ independent one-forms is called $q$-dimensional Pfaff system. Clearly, if $\alpha \in \mathcal{P}^{*}$ and $X \in \mathcal{P}$, then $\langle\alpha, X\rangle=0$. 
This allows to look at distributions as defined by a nonvanishing decomposable $p$ vector, or by a nonvanishing $(n-p)$-differential form. If $\mathcal{P} \Leftrightarrow\left\{X_{1}, X_{2}, \ldots, X_{p}\right\}$ then the $p$-vector field $\mathbb{P}=X_{1} \wedge X_{2} \wedge \cdots \wedge X_{p} \neq 0$ defines the distribution since at every point the representatives of $X_{i}, i=1, \ldots, p$ define the corresponding subspace $\Delta_{x} \subset T_{x}(M)$. An appropriate decomposable nonvanishing (n-p)-form $\Omega=\alpha^{1} \wedge \alpha^{2} \wedge \cdots \wedge \alpha^{n-p}$ such that $\left\langle\alpha^{m}, X_{i}\right\rangle=0$ also defines $\Delta_{x}(M)$.

A derivative of a distribution defined by the vector fields $\left(X_{1}, X_{2}, \ldots, X_{p}\right)$ is a new distribution $\mathcal{P}^{\prime}$ defined by the given $X_{i}, i=1,2, \ldots, p$ plus all Lie brackets $\left[X_{i}, X_{j}\right], i<j=1,2, \ldots, p$. In the same way higher derivatives of a given distribution can be defined.

It deserves noting, that the above definition of a distribution $\Delta$ on a manifold $M$ allows definite freedom in choosing appropriate local bases of $\Delta$ and allows also to form linear combinations of vectors in $\Delta$. But, on the other hand, it requires basis independence of all essential statements concerning $\Delta$.

\subsection{Morphisms of Distributions}

Let now $M$ and $N$ be two manifolds and $\Delta(M)$ and $\Delta(N)$ be two distributions on $M$ and $N$ respectively. Let $\varphi: M \rightarrow N$ be a smooth map. If $X_{x} \in \Delta_{x}(M)$, we consider its image $(\mathrm{d} \varphi)_{x}\left(X_{x}\right) \in T_{\varphi(x)}(N)$. If for every $x \in M$ every image of elements of $\Delta_{x}(M)$ is in $\Delta_{\varphi(x)}(N)$ we say that the coupe $(\varphi, \mathrm{d} \varphi)$ realize a morphism $\Delta(M) \rightarrow \Delta(N)$.

If $\varphi: M \rightarrow N$ is a diffeomorphism and $\Delta(M)$ and $\Delta(N)$ have the same dimension then the image $\operatorname{Im}(\Delta(M))=\left.(\varphi, \mathrm{d} \varphi)\right|_{\Delta(M)}$ of $\Delta(M)$ is a well defined distribution on $N$.

Correspondingly, every diffeomorphism $\varphi: M \rightarrow M$ sends a distribution on $M$ into another (in general) distribution on $M$.

\subsection{Integral Manifolds}

Similarly to the integral curves of vector fields, the concept of integral manifold of a $p$-dimensional distribution, or differential system, is introduced. Namely, a $p$ dimensional submanifold $V^{p}$ of $M$ is called integral manifold for the $p$-dimensional differential system $\mathcal{P}$, or for the $p$-dimensional distribution $\Delta^{p}(M)$, to which $\mathcal{P}$ belongs, if the tangent spaces of $V^{p}$ at every point coincide with the subspaces of the distribution $\Delta^{p}(M)$ at this point. In this case $V^{p}$ is called also integral manifold for the corresponding $(n-p)$-dimensional Pfaff system $\mathcal{P}^{*}$. If $\mathcal{P}$ admits at least one integral manifold, then it is called integrable. 
If through every point of $M$ there passes an integral manifold for $\mathcal{P}$, then $\mathcal{P}$ and $\mathcal{P}^{*}$ are called completely integrable.

A smooth function $f \in \mathcal{J}(M)$ is called first integral for $\mathcal{P}$ if $\mathrm{d} f \in \mathcal{P}^{*}$.

It can be shown that $\mathcal{P}$ is completely integrable iff it has

$$
n-p=\operatorname{codim}(\mathcal{P})=\operatorname{dim}\left(\mathcal{P}^{*}\right)
$$

functionally independent first integrals, i.e., locally $\mathcal{P}\left(\mathrm{d} f_{1}, \mathrm{~d} f_{2}, \ldots, \mathrm{d} f_{n-p}\right)$.

\subsection{Symmetries of Distributions}

A diffeomorphism $\varphi: M \rightarrow M$ is called a symmetry of the distribution $\Delta$ on $M$ if

$$
(\mathrm{d} \varphi)_{x}\left(\Delta_{x}\right)=\Delta_{\varphi(x)}, \quad x \in M .
$$

If $\Delta$ is defined by the linearly independent one-forms $\left\{\alpha^{1}, \alpha^{2}, \ldots, \alpha^{n-p}\right\}$ then we obtain the transformed by $\varphi$ one-forms $\left\{\varphi^{*} \alpha^{1}, \varphi^{*} \alpha^{2}, \ldots, \varphi^{*} \alpha^{n-p}\right\}$, which are also linearly independent, so, we have the relations

$$
\varphi^{*}\left(\alpha^{m}\right)=A_{n}^{m} \alpha^{n}
$$

where the matrix $A_{n}^{m}$ is non-degenerate at every point $x \in M$. These last relations may be written as follows:

$$
\varphi^{*}\left(\alpha^{m}\right) \wedge \alpha^{1} \wedge \alpha^{2} \wedge \cdots \wedge \alpha^{n-p}=0, \quad m=1,2, \ldots,(n-p) .
$$

i.e., without making use of the matrix $A_{n}^{m}$.

A vector field $X \in \mathfrak{X}(M)$ is called infinitesimal, or local symmetry of the distribution $\Delta$ if the corresponding flow $\varphi_{t}$ is a symmetry of $\Delta$. This is equivalent to say that

$$
L_{X}(\mathcal{P}) \subset \mathcal{P} \quad \text { or } \quad L_{X}\left(\mathcal{P}^{*}\right) \subset \mathcal{P}^{*} .
$$

In other words, the Lie derivative of every $Z \in \mathcal{P}$ is again in $\mathcal{P}$ and the Lie derivative of every $\alpha \in \mathcal{P}^{*}$ is again in $\mathcal{P}^{*}$.

Clearly, the set of infinitesimal symmetries of the distribution $\Delta$ is a Lie algebra (over $\mathbb{R}$ ), i.e., if $X, Y$ are infinitesimal symmetries of $\Delta$, then $X+Y, \lambda X, \lambda Y$ and $[X, Y]$ are also infinitesimal symmetries of $\Delta$.

There are two naturally identified subsets of all local (i.e., infinitesimal) symmetries of $\Delta$. The first subset, denoted by $\operatorname{char}(\mathcal{P})$, includes those vector fields which live in $\mathcal{P}$, and the second subset includes all the rest. Since if $X \in \operatorname{char}(\mathcal{P})$ can be represented as a linear combination of elements of $\mathcal{P}$ it is tangent to every integral manifold of $\Delta$. From the general relation $\left[L_{X}, i_{Y}\right]=i_{[X, Y]}$ it follows that the set 
$\operatorname{char}(\mathcal{P})$ is an ideal of the Lie algebra of all local symmetries of $\Delta$. Also, the set $\operatorname{char}(\mathcal{P})$ is a module over $\mathcal{J}(M)$.

The other subset of local symmetries, denoted by $\operatorname{shuf}(\mathcal{P})$, consists of vector fields that live outside $\mathcal{P}$ and are called shuffling symmetries of $\Delta$, the corresponding flows transform an integral manifold of $\Delta$ to another integral manifold of $\Delta$.

\subsection{Curvature of Distributions}

The concept of curvature of a distribution $\Delta^{p}(M)$ on a manifold $M$ is a local measure of the integrability properties of $\Delta^{p}(M)$, i.e., when $\Delta^{p}(M)$ admits an integral manifold. This problem is solved by the following theorem of Frobenius. In terms of symmetries this theorem can be formulated in the following way.

Frobenius theorem: A distribution $\Delta^{p}(M)$ is integrable iff all vector fields in $\mathcal{P}$ are symmetries of $\mathcal{P}$

If $\left\{X_{i}, i=1, \ldots, p\right\}$ are constituents of $\Delta^{p}(M)$ then this theorem says that $\Delta^{p}(M)$ is integrable only if all Lie brackets $\left[X_{i}, X_{j}\right], i<j=1, \ldots, p$ can be represented as $\mathcal{J}(M)$-linear combinations of $X_{i}, i=1, \ldots, p$

$$
\left[X_{i}, X_{j}\right]=f_{i j}^{k} X_{k}, \quad i<j, k=1, \ldots, p, \quad f_{i j}^{k} \in \mathcal{J}(M) .
$$

Therefore, if at least one of the following exterior products

$$
\left[X_{i}, X_{j}\right] \wedge X_{1} \wedge X_{2} \wedge \cdots \wedge X_{p}, \quad i, j=1, \ldots, p
$$

is different from zero, then $\Delta^{p}(M)$ is not integrable.

Let us see now how this criteria looks in terms of the codistribution $\mathcal{P}^{*}$. Recall that if $\left\{\alpha^{p+1}, \alpha^{p+2}, \ldots, \alpha^{n}\right\}$ are constituents of $\mathcal{P}^{*}$ then

$$
\left\langle\alpha^{m}, X_{i}\right\rangle=0, \quad m=(p+1), \ldots, n, \quad i=1,2, \ldots, p .
$$

Also, since all $\alpha^{m}$ are one-forms, then we obtain

$$
\mathbf{d} \alpha^{m}\left(X_{i}, X_{j}\right)=-\alpha^{m}\left(\left[X_{i}, X_{j}\right]\right), \quad m=(p+1), \ldots, n .
$$

Thus, if $\Delta^{p}(M)$ is integrable then the restriction of every $\mathbf{d} \alpha^{m}$ to $\Delta^{p}(M)$ has to be zero. This is equivalent to the requirements

$$
\mathbf{d} \alpha^{m} \wedge \alpha^{p+1} \wedge \alpha^{p+2} \cdots \wedge \alpha^{n}=0, \quad m=(p+1), \ldots, n .
$$

Locally, on every open subset $U \subset M$ we can introduce additional to $\Delta^{p}(U)$ distribution $\tilde{\Delta}^{n-p}(U)$, i.e., nonvanishing and linearly independent vector fields 
$\left\{X_{p+1}, X_{p+2}, \ldots X_{n}\right\}$, and additional to $\Delta_{n-p}^{*}(U)$ codistribution $\Delta_{p}^{*}(U)$, i.e., nonvanishing and linearly independent one-forms $\left\{\alpha^{1}, \alpha^{2}, \ldots, \alpha^{p}\right\}$ such that

$$
\left\langle\alpha^{i}, X_{j}\right\rangle=\delta_{j}^{i}, \quad i, j=1,2, \ldots, p \quad \text { and } \quad\left\langle\alpha^{m}, X_{s}\right\rangle=\delta_{s}^{m}, m, s=p+1, \ldots, n \text {. }
$$

Then $\Delta^{p}(U)$ and $\Delta^{\tilde{n}-p}(U)$ are called corresponding horizontal and vertical to each other.

Therefore, the nonintegrability of $\Delta^{p}(U)$ would mean

$$
\left(\left[X_{i}, X_{j}\right]-f_{i j}^{k} X_{k}\right) \notin \Delta^{p}(U), \quad i<j, k=1, \ldots, p
$$

or, equivalently

$$
\mathbf{d} \alpha^{m}+f_{s l}^{m} \alpha^{s} \wedge \alpha^{l} \neq 0, \quad m, s<l=p+1, \ldots, n .
$$

As an example, consider the manifold $\mathbb{R}^{3}$ (with the corresponding identification of forms and vector fields by the Euclidean metric) and a two-dimensional distribution defined by the one-form $\alpha$. The corresponding integrability condition looks like $\mathbf{d} \alpha \wedge \alpha=0$. In terms of vector analysis this condition is equivalent to $\vec{A} \cdot \operatorname{rot} \tilde{\mathrm{A}}=$ 0 . Hence, the nonintegrability of the two-dimensional distribution defined by $\alpha$ requires that $\vec{A}$ to be non-orthogonal to $\operatorname{rot} \tilde{\mathrm{A}}$, we recall that in hydrodynamics this quantity $\vec{A} \cdot \operatorname{rot} \tilde{\mathrm{A}}$ is called local helicity of the vector field $\vec{A}$.

We form now the following objects

$$
\Omega=-\mathbf{d} \alpha^{m} \otimes X_{m}, \quad m=p+1, \ldots, n
$$

and

$$
\tilde{\Omega}=-\mathbf{d} \alpha^{i} \otimes X_{i}, \quad i=1,2, \ldots, p .
$$

The restriction of $\Omega$ to $\Delta^{p}(U)$ is called curvature form for $\Delta^{p}(U)$, and the restriction of $\tilde{\Omega}$ to $\tilde{\Delta}^{n-p}(U)$ is called co-curvature form for $\Delta^{p}(U)$, or just curvature form for $\tilde{\Delta}^{n-p}(U)$.

It is seen that the restriction of $\Omega$ to $\Delta^{p}(U)$ is given by

$$
\begin{gathered}
\left.\Omega\right|_{\Delta^{p}(U)}\left(X_{i}, X_{j}\right)=-\mathbf{d} \alpha^{m}\left(X_{i}, X_{j}\right) X_{m}=\alpha^{m}\left(\left[X_{i}, X_{j}\right]\right) \\
i, j=1,2, \ldots, p, \quad m=p+1, \ldots, n
\end{gathered}
$$

and the restriction of $\tilde{\Omega}$ to $\tilde{\Delta}^{n-p}(U)$ is given by

$$
\begin{gathered}
\left.\tilde{\Omega}\right|_{\tilde{\Delta}^{n-p}(U)}\left(X_{m}, X_{n}\right)=-\mathbf{d} \alpha^{i}\left(X_{m}, X_{n}\right) X_{i}=\alpha^{i}\left(\left[X_{m}, X_{n}\right]\right) \\
i=1,2, \ldots, p, \quad m, n=p+1, \ldots, n .
\end{gathered}
$$

Clearly, $\Omega$ selects those Lie brackets in $\mathcal{P}(U)$ which 'stick out' of $\Delta^{p}(U)$ and $\tilde{\Omega}$ selects those Lie brackets in $\tilde{\mathcal{P}}(U)$ which 'stick out' of $\tilde{\Delta}^{n-p}(U)$. 


\subsection{Curvature in Terms of Projections in Tangent Bundle}

From the above we see that every distribution $\Delta^{p}(M)$ in $T_{M}$ defines a projection $P_{x} ; T_{x}(M) \rightarrow T_{x}(M), x \in M$, and inversely, every section $P$ of $T_{M} \otimes T_{M}^{*}$, i.e., $P \in \Lambda^{1}\left(M, T_{M}\right)$, with constant kernel: $\operatorname{ker}\left(P_{x}\right)=p$, defines a $p$-dimensional distribution $\Delta^{p}(M)$ on $M$ and corresponding $(n-p)$-codistribution $\Delta_{n-p}^{*}(M)$.

Let $P_{V}^{(1, p)}(x)$ projects on the subspace generated by $\left\{\frac{\partial}{\partial x^{1}}, \ldots, \frac{\partial}{\partial x^{p}}\right\}$, then the vertical subspace at $x \in M$ is the image of $P_{V}^{(1, p)}(x)$, or of the kernel of $P_{H}^{(1, p)}(x)=$ $\operatorname{Id}_{T M}-P_{V}^{(1, p)}(x)$. The local horizontal vector fields $X_{a}$ and vertical one-forms are $\alpha^{i}$ are given by

$$
X_{a}=\frac{\partial}{\partial x^{a}}+N_{a}^{i}(x) \frac{\partial}{\partial x^{i}}, \quad \alpha^{i}=\mathrm{d} x^{i}-N_{a}^{i}(x) \mathrm{d} x^{a} .
$$

The corresponding projections are

$$
\begin{aligned}
& P_{V}^{(1, p)}(x)=\alpha^{i} \otimes \frac{\partial}{\partial x^{i}}=\left[\mathrm{d} x^{i}-N_{a}^{i}(x) \mathrm{d} x^{a}\right] \otimes \frac{\partial}{\partial x^{i}} \\
& P_{H}^{(1, p)}(x)=\mathrm{d} x^{a} \otimes X_{a}=\mathrm{d} x^{a} \otimes\left(\frac{\partial}{\partial x^{a}}+N_{a}^{j}(x) \frac{\partial}{\partial x^{j}}\right) .
\end{aligned}
$$

Clearly, $P_{H}^{(1, p)}(x)$ projects on the subspace generated by $\frac{\partial}{\partial x^{a}}+N_{a}^{j} \frac{\partial}{\partial x^{j}}$, so, the horizontal subspace at $x \in M$ is the image of $P_{H}^{(1, p)}(x)$, or the kernel $P_{V}^{(1, p)}(x)$, and we have $\left\langle\alpha^{i}, X_{a}\right\rangle=0$.

If we want to call the subspace generated by $\left\{\frac{\partial}{\partial x^{p+1}}, \ldots, \frac{\partial}{\partial x^{n}}\right\}$ vertical, then the local horizontal vector fields $X_{i}$ and vertical one-forms are $\alpha^{a}$ are given by

$$
X_{i}=\frac{\partial}{\partial x^{i}}+N_{i}^{a}(x) \frac{\partial}{\partial x^{a}}, \quad \alpha^{a}=\mathrm{d} x^{a}-N_{i}^{a}(x) \mathrm{d} x^{i} .
$$

The corresponding projections should look like

$$
\begin{aligned}
& P_{V}^{(p+1, n)}(x)=\alpha^{a} \otimes \frac{\partial}{\partial x^{a}}=\left[\mathrm{d} x^{a}-N_{i}^{a}(x) \mathrm{d} x^{i}\right] \otimes \frac{\partial}{\partial x^{a}} \\
& P_{H}^{(p+1, n)}(x)=\mathrm{d} x^{i} \otimes X_{i}=\mathrm{d} x^{i} \otimes\left(\frac{\partial}{\partial x^{i}}+N_{i}^{a}(x) \frac{\partial}{\partial x^{a}}\right) .
\end{aligned}
$$

In both cases, of course, we have $P=P_{V} \oplus P_{H}$.

Definition 1. The projections in $T_{M}$ of constant rank are cold nonlinear, or general, connections. 
In general the curvature $\mathcal{R}_{P}$ and co-curvature $\tilde{\mathcal{R}}_{P}$ forms are given by

$$
\begin{aligned}
\mathcal{R}_{P}([,], X, Y)+\tilde{\mathcal{R}}_{P}([,], X, Y) & \\
& =P_{V}\left(\left[P_{H}(X), P_{H}(Y)\right]\right)+P_{H}\left(\left[P_{V}(X), P_{V}(Y)\right]\right) .
\end{aligned}
$$

Recalling the introduced curvature and co-curvature of a distribution as introduced in the previous subsection and identifying $\operatorname{Ker} P_{V}$ with $\Delta^{p}(M)$, and $\operatorname{Im} P_{V}$ with $\Delta^{n-p}(M)$ we can write

$$
\mathcal{R}_{P}=\left.\Omega\right|_{\Delta^{p}(M)}, \quad \tilde{\mathcal{R}}_{P}=\left.\Omega\right|_{\Delta^{n-p}(M)} .
$$

We are going now to see how these curvature forms look locally in terms of the projection components $N_{a}^{i}$, or $N_{i}^{a}$. In the first case we have to compute $\Omega_{a b}^{j}=\alpha^{j}\left(\left[X_{a}, X_{b}\right]\right), a<b$, and in the second case we have to compute $\Omega_{i j}^{a}=$ $\alpha^{a}\left(\left[X_{i}, X_{j}\right]\right), i<j$, where $i, j=1,2, \ldots, p, a, b=p+1, p+2, \ldots, n$

$$
\begin{aligned}
& \Omega_{a b}^{j}=\frac{\partial N_{b}^{j}}{\partial x^{a}}-\frac{\partial N_{a}^{j}}{\partial x^{b}}+N_{a}^{k} \frac{\partial N_{b}^{j}}{\partial x^{k}}-N_{b}^{k} \frac{\partial N_{a}^{j}}{\partial x^{k}} \\
& \Omega_{i j}^{a}=\frac{\partial N_{j}^{a}}{\partial x^{i}}-\frac{\partial N_{i}^{a}}{\partial x^{j}}+N_{i}^{b} \frac{\partial N_{j}^{a}}{\partial x^{b}}-N_{j}^{b} \frac{\partial N_{i}^{a}}{\partial x^{b}} .
\end{aligned}
$$

If we would like to obtain the two curvature forms directly from the corresponding vertical one-forms $\alpha^{i}, i=1,2, \ldots, p$, or from $\alpha^{a}, a=p+1, \ldots, n$, then we have to compute the corresponding horizontal projections of $\mathbf{d} \alpha^{i}$ and $\mathbf{d} \alpha^{a}$. Since $\alpha^{i}=$ $\mathrm{d} x^{i}-N_{a}^{i} \mathrm{~d} x^{a}$ then

$$
\mathbf{d} \alpha^{i}=-\mathbf{d}\left(N_{a}^{i} \mathrm{~d} x^{a}\right)=-\frac{\partial N_{a}^{i}}{\partial x^{j}} \mathrm{~d} x^{j} \wedge \mathrm{d} x^{a}-\frac{\partial N_{a}^{i}}{\partial x^{b}} \mathrm{~d} x^{b} \wedge \mathrm{d} x^{a} .
$$

Now, in this case we have that $\mathrm{d} x^{a}$ are horizontal, so we have to find the horizontal part of $\mathrm{d} x^{j}$. Since the restriction of $\mathrm{d} x^{j}$ to $\Delta^{p}(M)$ is equal to $N_{a}^{j} \mathrm{~d} x^{a}$, we obtain

$$
\left.\mathbf{d} \alpha^{i}\right|_{\Delta^{n-p}(M)}=-\left(\frac{\partial N_{a}^{i}}{\partial x^{b}}-\frac{\partial N_{b}^{i}}{\partial x^{a}}+\frac{\partial N_{a}^{i}}{\partial x^{j}} N_{b}^{j}-\frac{\partial N_{b}^{i}}{\partial x^{j}} N_{a}^{j}\right) \mathrm{d} x^{b} \wedge \mathrm{d} x^{a}, \quad b<a .
$$

In the same way for the second case we obtain

$$
\left.\mathbf{d} \alpha^{a}\right|_{\Delta^{p}(M)}=-\left(\frac{\partial N_{i}^{a}}{\partial x^{j}}-\frac{\partial N_{j}^{a}}{\partial x^{i}}+\frac{\partial N_{i}^{a}}{\partial x^{b}} N_{j}^{b}-\frac{\partial N_{j}^{a}}{\partial x^{b}} N_{i}^{b}\right) \mathrm{d} x^{j} \wedge \mathrm{d} x^{i}, \quad j<i .
$$

Hence, for $\left.\Omega\right|_{\Delta^{p}(M)}$ and $\left.\Omega\right|_{\Delta^{n-p}}$ we obtain respectively

$$
\begin{aligned}
\left.\Omega\right|_{\Delta^{p}(M)} & =-\left(\left.\mathbf{d} \alpha^{a}\right|_{\Delta^{p}(M)}\right) \otimes \frac{\partial}{\partial x^{a}} \\
= & \left(\frac{\partial N_{i}^{a}}{\partial x^{j}}-\frac{\partial N_{j}^{a}}{\partial x^{i}}+\frac{\partial N_{i}^{a}}{\partial x^{b}} N_{j}^{b}-\frac{\partial N_{j}^{a}}{\partial x^{b}} N_{i}^{b}\right) \mathrm{d} x^{j} \wedge \mathrm{d} x^{i} \otimes \frac{\partial}{\partial x^{a}}, \quad j<i
\end{aligned}
$$




$$
\begin{aligned}
& \left.\Omega\right|_{\Delta^{n-p}(M)}=-\left(\left.\mathbf{d} \alpha^{i}\right|_{\Delta^{n-p}(M)}\right) \otimes \frac{\partial}{\partial x^{i}} \\
& \quad=\left(\frac{\partial N_{a}^{i}}{\partial x^{b}}-\frac{\partial N_{b}^{i}}{\partial x^{a}}+\frac{\partial N_{a}^{i}}{\partial x^{j}} N_{b}^{j}-\frac{\partial N_{b}^{i}}{\partial x^{j}} N_{a}^{j}\right) \mathrm{d} x^{b} \wedge \mathrm{d} x^{a} \otimes \frac{\partial}{\partial x^{i}}, \quad b<a .
\end{aligned}
$$

\section{Physical Interpretation}

An integrable distribution $\Delta^{p}$ on $M$ may contain many non-integrable subdistributions $\Delta_{1}^{p_{1}}, \Delta_{2}^{p_{2}}, \ldots, \quad p_{1}, p_{2}, \ldots<p$. Clearly, the corresponding curvature forms $\Omega_{1}^{p_{1}}, \Omega_{2}^{p_{2}}, \ldots$ of these subdistributions do NOT take values outside $\Delta^{p}$, so such a picture of available "intercommunication" between subdistributions of a higher dimensional integrable distribution by means of their curvature forms suggests the idea to try this geometrical approach as a possible model of a timestable continuous physical system built of relatively time-stable and continuously time-recognizable subsystems existing through some permanent interaction, i.e., through a permanent energy-momentum inter-exchange.

We formulate the basic assumptions:

- Any physical system with a dynamical structure is characterized by some internal energy-momentum redistributions, i.e., identifiable internal energymomentum fluxes, during evolution.

- Any time-stable (i.e., compatible) system of energy-momentum fluxes can be represented mathematically (directly or indirectly) by a an appropriate completely integrable distribution $\Delta$ on a manifold.

- A shuffling local symmetry $\zeta$ of a completely integrable distribution $\Delta=$ $\left\{X_{1}, \ldots, X_{p}\right\}$ may be considered to define the allowed space-time propagation of the corresponding physical system.

- The two-dimensional subdistributions $\left\{\zeta, X_{i}\right\}, i=1,2, \ldots, p$, defined by the local shuffling symmetry $\zeta$ and any of the constituents $X_{i} \in \Delta$ may be considered as mathematical models of the elementary structural time-stable subsystems of the physical system considered.

- The time-stability of the physical system of interacting energy-momentum fluxes can be considered mathematically in terms of the NON-integrability of these two-dimensional subdistributions $\left\{\zeta, X_{i}\right\}$, and the corresponding curvature forms $\Omega_{i}=\Omega_{\left(\zeta, X_{i}\right)}$ to be considered as the main interaction (i.e., energy-momentum transfer) agents between any two such subsystems. 
- The projection of $\Omega_{i}\left(X_{i}, \zeta\right)$ on the corresponding two-dimensional volume form of any subdistribution $\left\{\zeta, X_{j}\right\}, j \neq i$, to be considered as the transferred energy-momentum from $\left\{\zeta, X_{i}\right\}$ to $\left\{\zeta, X_{j}\right\}$.

Classically, this projection corresponds to the local flow of the vector field $\Omega_{i}\left(X_{i}, \zeta\right)$ across the two-surface represented locally by the corresponding volume two-form.

Shortly speaking, if $\Delta_{1}$ and $\Delta_{2}$ characterize two locally interacting physical systems, (i.e., physical fields), or two locally interacting subsystems of a larger physical system, it seems reasonable to assume as a working tool the following geometrization of the concept of local physical interaction:

Two nonintegrable distributions $\Delta_{1}$ and $\Delta_{2}$ on a manifold will be said to interact infinitesimally, or locally, if some of the nonzero values of the corresponding two curvature forms $\Omega_{1} / \Omega_{2}$ live respectively in $\Delta_{2} / \Delta_{1}$.

In this way, since any physical interaction between two physical field systems is necessarily accompanied with available energy-momentum exchange between them, we could understand it mathematically as nonintegrability of each of the two corresponding distributions. This interaction could be naturally measured directly or indirectly by the corresponding curvatures.

For example, if $\Delta$ is an integrable three-dimensional distribution represented by the set of vector fields $\left(X_{1}, X_{2}, X_{3}\right)$ then we may have, in general, three different non-integrable, i.e., geometrically interacting two-dimensional subdistributions $\left(X_{1}, X_{2}\right),\left(X_{1}, X_{3}\right)$ and $\left(X_{2}, X_{3}\right)$. Finally, some interaction with the outside world can be described by curvatures of distributions (and their subdistributions) in which elements of $\Delta$ and vector fields outside $\Delta$ are involved (such processes will not be considered here).

In other words we launch the general idea to consider the concept of Frobenius curvature as a natural and universal mathematical tool for describing local physical interaction between/among the relatively stable subsystems of the physical world.

Following this principle, the Frobenius curvature appears as appropriate mathematical tool describing formally the possible ability two continuous physical systems to recognize each other as physically interacting partners. More comments on this subject can found in [3].

\section{The Notion of Photon-like Object (PhLO)}

We introduce the following physical notion about PhLO [3] 


\section{PhLO are real massless time-stable physical objects with an intrinsically compatible translational-rotational dynamical structure.}

We give now some explanatory comments, beginning with the term real. First we emphasize that this term means that we consider PhLO as really existing physical objects, not as appropriate and helpful but imaginary (theoretical) entities. Accordingly, PhLO necessarily carry energy-momentum, otherwise, they could hardly be detected by physical means. Second, PhLO can undoubtedly be created and destroyed, so, no point-like and infinite models are reasonable: point-like objects are assumed to have no structure, so they can not be destroyed since there is no available structure to be destroyed. Creation of infinite physical objects (e.g. plane waves) requires infinite quantity of energy to be transformed from one kind to another during finite time-periods, which seems also unreasonable. Accordingly, $\mathrm{PhLO}$ are spatially finite and have to be modelled like such ones, which is the only possibility to be consistent with their 'created-destroyed' nature. It seems hardly reasonable to believe that PhLO can not be created and destroyed, and that spatially infinite and indestructible physical objects may exist at all. Third, 'spatially finite' implies that PhLO may carry only finite values of physical (conservative or non-conservative) quantities. In particular, the most universal physical quantity seems to be the energy-momentum, so the model must allow finite integral values of energy-momentum to be carried by the corresponding solutions. Fourth, 'spatially finite' means also that $\mathrm{PhLO}$ propagate, i.e., they do not 'move' like classical particles along trajectories, therefore, partial differential equations should be used to describe their evolution in time.

The term massless characterizes physically the way of propagation in terms of appropriate dynamical quantities: the integral four-momentum $p$ of a PhLO should satisfy the relation $p_{\mu} p^{\mu}=0$, meaning that its integral energy-momentum vector must be isotropic, i.e., to have zero module with respect to Minkowski pseudometric in $\mathbb{R}^{4}$. If the object considered has spatial and time-stable structure, so that the translational velocity of every point where the corresponding field functions are different from zero must be equal to $c$, we have in fact null direction in the spacetime intrinsically determined by a $\mathrm{PhLO}$. Such a direction is formally defined by a null vector field $\bar{\zeta}, \bar{\zeta}^{2}=0$. The integral trajectories of this vector field are isotropic (or null) straight lines as is traditionally assumed in physics, except in presence of gravity. It follows that with every $\mathrm{PhLO}$ a null straight line direction is necessarily associated, so, canonical coordinates $\left(x^{1}, x^{2}, x^{3}, x^{4}\right)=(x, y, z, \xi=c t)$ on $\mathbb{R}^{4}$ may be chosen such that in the corresponding coordinate frame $\bar{\zeta}$ to have only two non-zero components of magnitude $1: \bar{\zeta}^{\mu}=(0,0,-\varepsilon, 1)$, where $\varepsilon= \pm 1$ accounts for the two directions along the coordinate $z$ (further such a coordinate system will be called $\bar{\zeta}$-adapted and will be of main usage). It seems important to emphasize 
that our PhLO propagates as a whole along the $\bar{\zeta}$-direction, so the corresponding energy-momentum tensor field $T_{\mu \nu}(x, y, z, \xi)$ of the model must satisfy the corresponding local isotropy condition, namely, $T_{\mu \nu} T^{\mu \nu}=0$ (summation over the repeated indices is throughout used). All this means also, that the vector field $\bar{\zeta}$ should be considered as a shuffling local symmetry of a PhLO.

The term translational-rotational means that besides the translational component along $\bar{\zeta}$, the propagation necessarily demonstrates some rotational (in the general sense of this concept) component in such a way that both components are compatible and exist simultaneously, and this is an intrinsic property. It seems reasonable to expect that such kind of dynamical behavior should require some distinguished spatial shapes. Moreover, if the Planck relation $E=h \nu$ must be respected throughout the evolution, the rotational component of propagation should have time-periodical nature with time period $T=\nu^{-1}=h / E=$ const, and one of the two possible, left or right, orientations. It seems reasonable also to expect spatial periodicity of $\mathrm{PhLO}$, which somehow to be related to the time periodicity.

The term dynamical structure means that the propagation is supposed to be necessarily accompanied by an internal energy-momentum redistribution, which may be considered in the model as energy-momentum exchange between (or among) some appropriately defined subsystems. It could also mean that $\mathrm{PhLO}$ live in a dynamical harmony with the outside world, i.e., any outside directed energy-momentum flow should be accompanied by a parallel inside directed energy-momentum flow.

\section{PhLO Dynamical Structure in Terms of Frobenius Curvature}

We consider the Minkowski space-time $M=\left(\mathbb{R}^{4}, \eta\right)$ with $\operatorname{sign}(\eta)=(-,-,-,+)$ related to the standard coordinates $\left(x^{1}, x^{2}, x^{3}, x^{4}\right)=(x, y, z, \xi=c t)$, the natural volume form $\omega_{o}=\sqrt{|\eta|} \mathrm{d} x^{1} \wedge \mathrm{d} x^{2} \wedge \mathrm{d} x^{3} \wedge \mathrm{d} x^{4}=\mathrm{d} x \wedge \mathrm{d} y \wedge \mathrm{d} z \wedge \mathrm{d} \xi$, and the Hodge star $*$ defined by $\alpha \wedge * \beta=-\eta(\alpha, \beta) \omega_{0}$. We recall the reader that the Hodge $*$ introduces complex structure in $\Lambda^{2}(M)$ [4]. In view of our concept of PhLO we introduce the null vector field $\bar{\zeta}, \bar{\zeta}^{2}=0$, which in the $\bar{\zeta}$-adapted coordinates (throughout used further) is assumed to look as follows

$$
\bar{\zeta}=-\varepsilon \frac{\partial}{\partial z}+\frac{\partial}{\partial \xi}, \quad \varepsilon= \pm 1 .
$$

Let us denote the corresponding to $\bar{\zeta}$ completely integrable three-dimensional Pfaff system by $\Delta^{*}(\bar{\zeta})$. Thus, $\Delta^{*}(\bar{\zeta})$ can be generated by any three linearly independent one-forms $\left(\alpha_{1}, \alpha_{2}, \alpha_{3}\right)$ which annihilate $\bar{\zeta}$, i.e.,

$$
\alpha_{1}(\bar{\zeta})=\alpha_{2}(\bar{\zeta})=\alpha_{3}(\bar{\zeta})=0, \quad \alpha_{1} \wedge \alpha_{2} \wedge \alpha_{3} \neq 0 .
$$


Instead of $\left(\alpha_{1}, \alpha_{2}, \alpha_{3}\right)$ we introduce the notation $\left(A, A^{*}, \zeta\right)$ and define $\zeta$ to be the $\eta$-corresponding one-form to $\bar{\zeta}$ :

$$
\zeta=\varepsilon \mathrm{d} z+\mathrm{d} \xi, \quad \text { so } \quad\langle\zeta, \bar{\zeta}\rangle=0
$$

where $\langle$,$\rangle is the coupling between forms and vectors.$

Now, since $\zeta$ is closed, it defines one-dimensional completely integrable Pfaff system, so, we have the corresponding completely integrable distribution $\left(\bar{A}, \bar{A}^{*}, \bar{\zeta}\right)$ : $\langle\zeta, \bar{A}\rangle=\left\langle\zeta, \bar{A}^{*}\right\rangle=0$. We shall restrict our further study to PhLO of electromagnetic nature according to the following

Definition 2. We shall call a PhLO electromagnetic if the following conditions hold

1. the vector fields $\left(\bar{A}, \bar{A}^{*}\right)$ have no components along $\bar{\zeta}$

2. $\left(\bar{A}, \bar{A}^{*}\right)$ are $\eta$-corresponding to $\left(A, A^{*}\right)$ respectively

3. $\left\langle A, \bar{A}^{*}\right\rangle=0,\langle A, \bar{A}\rangle=\left\langle A^{*}, \bar{A}^{*}\right\rangle$.

Remark 3. These relations formalize knowledge from Classical Electrodynamics (CED). In fact, our vector fields $\left(\bar{A}, \bar{A}^{*}\right)$ are meant to represent what we call in $C E D$ electric and magnetic components of a free time-dependent electromagnetic field, where, as is well known, the translational propagation of the field energymomentum along a fixed null direction with the velocity ' $c$ ' is possible only if the two invariants $I_{1}=\mathbf{B}^{2}-\mathbf{E}^{2}$ and $I_{2}=2 \mathbf{E}$. $\mathbf{B}$ are zero, because only in such a case the electromagnetic energy-momentum tensor $T_{\mu \nu}$ satisfies $T_{\mu \nu} T^{\mu \nu}=0$ and has unique null eigen direction. So it seems naturally to consider this property as intrinsic for the field and to choose it as a starting point. Moreover, in such a case the relation $T_{\mu \nu} T^{\mu \nu}=\left(I_{1}\right)^{2}+\left(I_{2}\right)^{2}=0$ is equivalent to $\mathbf{E}^{2}+\mathbf{B}^{2}=2|\mathbf{E} \times \mathbf{B}|$ and this relation shows that this is the only case when the field momentum can not be made equal to zero by means of frame change.

From the above conditions it follows that in the $\bar{\zeta}$-adapted coordinate system we have

$$
\begin{array}{lll}
A=u \mathrm{~d} x+p \mathrm{~d} y, & A^{*}=-\varepsilon p \mathrm{~d} x+\varepsilon u \mathrm{~d} y \\
\bar{A}=-u \frac{\partial}{\partial x}-p \frac{\partial}{\partial y}, & \bar{A}^{*}=\varepsilon p \frac{\partial}{\partial x}-\varepsilon u \frac{\partial}{\partial y}
\end{array}
$$

where $\varepsilon= \pm 1$, and $(u, p)$ are two smooth functions on $M$.

The completely integrable three-dimensional Pfaff system $\left(A, A^{*}, \zeta\right)$ contains three two-dimensional subsystems: $\left(A, A^{*}\right),(A, \zeta)$ and $\left(A^{*}, \zeta\right)$. We have the following 
Proposition 4. The following relations hold

$$
\begin{gathered}
\mathbf{d} A \wedge A \wedge A^{*}=0, \quad \mathbf{d} A^{*} \wedge A^{*} \wedge A=0 \\
\mathbf{d} A \wedge A \wedge \zeta=\varepsilon\left[u\left(p_{\xi}-\varepsilon p_{z}\right)-p\left(u_{\xi}-\varepsilon u_{z}\right)\right] \omega_{o} \\
\mathbf{d} A^{*} \wedge A^{*} \wedge \zeta=\varepsilon\left[u\left(p_{\xi}-\varepsilon p_{z}\right)-p\left(u_{\xi}-\varepsilon u_{z}\right)\right] \omega_{o} .
\end{gathered}
$$

Proof: Immediately verified.

These relations say that the two-dimensional Pfaff system $\left(A, A^{*}\right)$ is completely integrable for any choice of the two functions $(u, p)$, while the two two-dimensional Pfaff systems $(A, \zeta)$ and $\left(A^{*}, \zeta\right)$ are NOT completely integrable in general, and the same curvature factor

$$
\mathbf{R}=u\left(p_{\xi}-\varepsilon p_{z}\right)-p\left(u_{\xi}-\varepsilon u_{z}\right)
$$

determines their nonintegrability.

Correspondingly, the three-dimensional completely integrable distribution (or differential system) $\Delta(\bar{\zeta})$ contains three two-dimensional subsystems $\left(\bar{A}, \bar{A}^{*}\right),(\bar{A}, \bar{\zeta})$ and $\left(\bar{A}^{*}, \bar{\zeta}\right)$. We have the

Proposition 5. The following relations hold

$$
\begin{gathered}
{\left[\bar{A}, \bar{A}^{*}\right] \wedge \bar{A} \wedge \bar{A}^{*}=0} \\
{[\bar{A}, \bar{\zeta}]=\left(u_{\xi}-\varepsilon u_{z}\right) \frac{\partial}{\partial x}+\left(p_{\xi}-\varepsilon p_{z}\right) \frac{\partial}{\partial y}} \\
{\left[\bar{A}^{*}, \bar{\zeta}\right]=-\varepsilon\left(p_{\xi}-\varepsilon p_{z}\right) \frac{\partial}{\partial x}+\varepsilon\left(u_{\xi}-\varepsilon u_{z}\right) \frac{\partial}{\partial y} .}
\end{gathered}
$$

Proof: Immediately verified.

From these last relations it follows that the distribution $\left(\bar{A}, \bar{A}^{*}\right)$ is completely integrable, and it can be easily shown that the two distributions $(\bar{A}, \bar{\zeta})$ and $\left(\bar{A}^{*}, \bar{\zeta}\right)$ would be completely integrable only if the same curvature factor

$$
\mathbf{R}=u\left(p_{\xi}-\varepsilon p_{z}\right)-p\left(u_{\xi}-\varepsilon u_{z}\right)
$$

is zero (the elementary proof is omitted).

As it should be, the two projections

$$
\left\langle A,\left[\bar{A}^{*}, \bar{\zeta}\right]\right\rangle=-\left\langle A^{*},[\bar{A}, \bar{\zeta}]\right\rangle=-\varepsilon u\left(p_{\xi}-\varepsilon p_{z}\right)+\varepsilon p\left(u_{\xi}-\varepsilon u_{z}\right)=-\varepsilon \mathbf{R}
$$


are nonzero and give (up to a sign) the same factor $\mathbf{R}$. The same curvature factor appears, of course, as coefficient in the exterior products $\left[\bar{A}^{*}, \bar{\zeta}\right] \wedge \bar{A}^{*} \wedge \bar{\zeta}$ and $[\bar{A}, \bar{\zeta}] \wedge \bar{A} \wedge \bar{\zeta}$. In fact, we obtain

$\left[\bar{A}^{*}, \bar{\zeta}\right] \wedge \bar{A}^{*} \wedge \bar{\zeta}=-[\bar{A}, \bar{\zeta}] \wedge \bar{A} \wedge \bar{\zeta}=-\varepsilon \mathbf{R} \frac{\partial}{\partial x} \wedge \frac{\partial}{\partial y} \wedge \frac{\partial}{\partial z}+\mathbf{R} \frac{\partial}{\partial x} \wedge \frac{\partial}{\partial y} \wedge \frac{\partial}{\partial \xi}$

On the other hand, for the other two projections we obtain

$$
\langle A,[\bar{A}, \bar{\zeta}]\rangle=\left\langle A^{*},\left[\bar{A}^{*}, \bar{\zeta}\right]\right\rangle=\frac{1}{2}\left[\left(u^{2}+p^{2}\right)_{\xi}-\varepsilon\left(u^{2}+p^{2}\right)_{z}\right] .
$$

Clearly, the last relation may be put in terms of the Lie derivative $L_{\bar{\zeta}}$ as

$$
\frac{1}{2} L_{\bar{\zeta}}\left(u^{2}+p^{2}\right)=-\frac{1}{2} L_{\bar{\zeta}}\langle A, \bar{A}\rangle=-\left\langle A, L_{\bar{\zeta}} \bar{A}\right\rangle=-\left\langle A^{*}, L_{\bar{\zeta}} \bar{A}^{*}\right\rangle .
$$

Remark 6. Further we shall denote $\sqrt{u^{2}+p^{2}} \equiv \phi$.

Proposition 7. There is a function $\psi(u, p)$ such, that

$$
L_{\bar{\zeta}} \psi=\frac{u\left(p_{\xi}-\varepsilon p_{z}\right)-p\left(u_{\xi}-\varepsilon u_{z}\right)}{\phi^{2}}=\frac{\mathbf{R}}{\phi^{2}} .
$$

Proof: It is immediately verified that $\psi=\arctan \frac{p}{u}$ is such one.

We note that the function $\psi$ has a natural interpretation of phase because of the easily verified now relations $u=\phi \cos \psi, p=\phi \sin \psi$, and $\phi$ acquires the status of amplitude, i.e., energy density. Since the transformation $(u, p) \rightarrow(\phi, \psi)$ is non-degenerate this allows to work with the two functions $(\phi, \psi)$ instead of $(u, p)$. From Proposition 3 we have

$$
\mathbf{R}=\phi^{2} L_{\bar{\zeta}} \psi=\phi^{2}\left(\psi_{\xi}-\varepsilon \psi_{z}\right) \rightarrow L_{\bar{\zeta}} \psi=\frac{\mathbf{R}}{T\left(\partial_{\xi}, \partial_{\xi}\right)}=\frac{* \varepsilon(\mathbf{d} A \wedge A \wedge \zeta)}{T\left(\partial_{\xi}, \partial_{\xi}\right)}
$$

where $T\left(\partial_{\xi}, \partial_{\xi}\right)$ is the coordinate-free definition of the energy density.

This last formula shows something very important: at any $\phi \neq 0$ the curvature $\mathbf{R}$ will NOT be zero only if $L_{\bar{\zeta}} \psi \neq 0$, which admits in principle availability of rotation. In fact, lack of rotation would mean that $\phi$ and $\psi$ are running waves along $\bar{\zeta}$. The relation $L_{\bar{\zeta}} \psi \neq 0$ means, however, that rotational properties are possible in general, and some of these properties are carried by the phase $\psi$. It follows that in such a case the translational component of propagation along $\bar{\zeta}$ (which is supposed to be available) must be determined essentially, and most probably entirely, 
by $\phi$. In particular, we could expect the relation $L_{\bar{\zeta}} \phi=0$ to hold, and if this happens, then the rotational component of propagation will be represented entirely by the phase $\psi$, and, more specially, by the curvature factor $\mathbf{R} \neq 0$, so, the objects we are going to describe may have compatible translational-rotational dynamical structure. Finally, this relation defines, in fact, the phase function $\psi$.

We are going now to represent some relations, analogical to the energy-momentum relations in classical electrodynamics, determined by some two-form $F$, in terms of the Frobenius curvatures given above.

The two nonintegrable Pfaff systems $(A, \zeta)$ and $\left(A^{*}, \zeta\right)$ carry two volume twoforms

$$
G=A \wedge \zeta \quad \text { and } \quad G^{*}=A^{*} \wedge \zeta
$$

and the two corresponding distributions define the two-vectors

$$
\bar{G}=\bar{A} \wedge \bar{\zeta} \quad \text { and } \quad \bar{G}^{*}=\bar{A}^{*} \wedge \bar{\zeta} .
$$

Making use now of the Hodge $*$-operator, we can verify the relation: $G^{*}=* G$. Now $G$ and $\bar{G}^{*}$ define the $(1,1)$-tensor, called stress-energy-momentum tensor $T_{\mu}^{\nu}$, according to the rule

$$
T_{\mu}^{\nu}=-\frac{1}{2}\left[G_{\mu \sigma} \bar{G}^{\nu \sigma}+\left(G^{*}\right)_{\mu \sigma}\left(\bar{G}^{*}\right)^{\nu \sigma}\right]
$$

and the divergence of this tensor field can be represented in the form

$$
\nabla_{\nu} T_{\mu}^{\nu}=[i(\bar{G}) \mathbf{d} G]_{\mu}+\left[i\left(\bar{G}^{*}\right) \mathbf{d} G^{*}\right]_{\mu}
$$

where $\bar{G}$ and $\bar{G}^{*}$ coincide with the metric-corresponding contravariant tensor fields, and $i(\bar{G})=i(\bar{\zeta}) \circ i(\bar{A}), i\left(\bar{G}^{*}\right)=i(\bar{\zeta}) \circ i\left(\bar{A}^{*}\right), i(X)$ is the standard insertion operator in the exterior algebra of differential forms on $\mathbb{R}^{4}$ defined by the vector field $X$. So, we shall need the quantities

$$
i(\bar{G}) \mathbf{d} G, \quad i\left(\bar{G}^{*}\right) \mathbf{d} G^{*}, \quad i\left(\bar{G}^{*}\right) \mathbf{d} G, \quad i(\bar{G}) \mathbf{d} G^{*} .
$$

Having in view the explicit expressions for $A, A^{*}, \zeta, \bar{A}, \overline{A^{*}}$ and $\bar{\zeta}$ we obtain

$$
i(\bar{G}) \mathbf{d} G=i\left(\bar{G}^{*}\right) \mathbf{d} G^{*}=\frac{1}{2} L_{\bar{\zeta}}\left(\phi^{2}\right) \cdot \zeta
$$

and

$$
\begin{gathered}
i\left(\bar{G}^{*}\right) \mathbf{d} G=-i(\bar{G}) \mathbf{d} G^{*} \\
=\left[u\left(p_{\xi}-\varepsilon p_{z}\right)-p\left(u_{\xi}-\varepsilon u_{z}\right)\right] d z+\varepsilon\left[u\left(p_{\xi}-\varepsilon p_{z}\right)-p\left(u_{\xi}-\varepsilon u_{z}\right)\right] \mathrm{d} \xi=\varepsilon \mathbf{R} \zeta .
\end{gathered}
$$


If $F$ and $H$ are correspondingly two and three forms on $M$ we have the relation

$$
*(F \wedge * H)=i(\bar{F}) H=F^{\mu \nu} H_{\mu \nu \sigma} \mathrm{d} x^{\sigma}, \quad \mu<\nu .
$$

Therefore, since $G^{*}=* G$,

$$
i\left(\bar{G}^{*}\right) \mathbf{d} G=-*(\delta * G \wedge * G) \quad i(\bar{G}) \mathbf{d} G^{*}=*(\delta G \wedge G)
$$

and

$$
\delta * G \wedge * G=\delta G \wedge G=\varepsilon \mathbf{R} * \zeta .
$$

In the following formulae we must keep in mind the relations $\mathbf{d} \zeta=0,\left\langle A, \bar{A}^{*}\right\rangle=$ $\left\langle A^{*}, \bar{A}\right\rangle=\left\langle\zeta, \bar{A}^{*}\right\rangle=\langle\zeta, \bar{A}\rangle=0$, and $(\bar{A})^{2}=\left(\bar{A}^{*}\right)^{2}=\langle A, \bar{A}\rangle=\left\langle A^{*}, \bar{A}^{*}\right\rangle=$ $-\left(u^{2}+p^{2}\right)=-\Phi^{2}=-|A|^{2}=-\left|A^{*}\right|^{2}=-|\bar{A}|^{2}=-\left|\bar{A}^{*}\right|^{2}$.

In view of these formulae and the required duality in the definition of the curvature form the two distributions $(\bar{A}, \bar{\zeta})$ and $\left(\bar{A}^{*}, \bar{\zeta}\right)$ determine the following two curvature forms $\Omega$ and $\Omega^{*}$

$\Omega=-\mathbf{d} \frac{-A^{*}}{\left|A^{*}\right|} \otimes \frac{\bar{A}^{*}}{\left|\bar{A}^{*}\right|}=\mathbf{d} \frac{A^{*}}{\left|A^{*}\right|} \otimes \frac{\bar{A}^{*}}{\left|\bar{A}^{*}\right|}, \quad \Omega^{*}=-\mathbf{d} \frac{-A}{|A|} \otimes \frac{\bar{A}}{|\bar{A}|}=\mathbf{d} \frac{A}{|A|} \otimes \frac{\bar{A}}{|\bar{A}|}$.

Denoting $Z_{\Omega} \equiv \Omega(\bar{A}, \bar{\zeta}), Z_{\Omega}^{*} \equiv \Omega\left(\bar{A}^{*}, \bar{\zeta}\right), Z_{\Omega^{*}} \equiv \Omega^{*}(\bar{A}, \bar{\zeta})$ and $Z_{\Omega^{*}}^{*} \equiv \Omega^{*}\left(\bar{A}^{*}, \bar{\zeta}\right)$ we obtain

$$
\begin{aligned}
2 Z_{\Omega} & =-\frac{\varepsilon \mathbf{R}}{\phi^{2}} \bar{A}^{*}, & Z_{\Omega}^{*} & =-\frac{\bar{A}^{*}}{2 \phi^{2}} L_{\bar{\zeta}}\left(\phi^{2}\right) \\
Z_{\Omega^{*}} & =-\frac{\bar{A}}{2 \phi^{2}} L_{\bar{\zeta}}\left(\phi^{2}\right), & Z_{\Omega^{*}}^{*} & =\frac{\varepsilon \mathbf{R}}{\phi^{2}} \bar{A} .
\end{aligned}
$$

The following relations express the connection between the curvatures and the energy-momentum characteristics

$$
\begin{aligned}
& i\left(Z_{\Omega}\right)(A \wedge \zeta)=0, i\left(Z_{\Omega}\right)\left(A^{*} \wedge \zeta\right)=\varepsilon \mathbf{R} \cdot \zeta=-i(\bar{G}) \mathbf{d} G^{*}=i\left(\bar{G}^{*}\right) \mathbf{d} G \\
& i\left(Z_{\Omega^{*}}\right)\left(A^{*} \wedge \zeta\right)=0, i\left(Z_{\Omega^{*}}^{*}\right)(A \wedge \zeta)=-\varepsilon \mathbf{R} \cdot \zeta=i(\bar{G}) \mathbf{d} G^{*}=-i\left(\bar{G}^{*}\right) \mathbf{d} G \\
& i\left(Z_{\Omega}^{*}\right)(A \wedge \zeta)=0, \quad i\left(Z_{\Omega}^{*}\right)\left(A^{*} \wedge \zeta\right)=\frac{1}{2} L_{\bar{\zeta}}\left(\phi^{2}\right) \cdot \zeta=i(\bar{G}) \mathbf{d} G=i\left(\bar{G}^{*}\right) \mathbf{d} G^{*} \\
& i\left(Z_{\Omega^{*}}^{*}\right)\left(A^{*} \wedge \zeta\right)=0, \quad i\left(Z_{\Omega^{*}}\right)(A \wedge \zeta)=\frac{1}{2} L_{\bar{\zeta}}\left(\phi^{2}\right) \cdot \zeta=i(\bar{G}) \mathbf{d} G=i\left(\bar{G}^{*}\right) \mathbf{d} G^{*} .
\end{aligned}
$$

Recalling that in this null-field case we have always fulfilled the equality

$$
G_{\mu \sigma} G^{\nu \sigma}=(* G)_{\mu \sigma}(* G)^{\nu \sigma}
$$


it follows from the above relations and from the local conservation law $\nabla_{\nu} T_{\mu}^{\nu}=0$ that the two subsystems of our $\mathrm{PhLO}$, described by $G$ and $* G$, live in a dynamical equilibrium, i.e., each loses as much energy-momentum as it gains locally. So, we obtain the equations

$$
i(\bar{G}) \mathbf{d} G=0, \quad i\left(\bar{G}^{*}\right) \mathbf{d} G^{*}=0, \quad i\left(\bar{G}^{*}\right) \mathbf{d} G+i(\bar{G}) \mathbf{d} G^{*}=0
$$

describing the dynamical behaviour of PhLO.

Finally, the local representative of the spin properties of a $\mathrm{PhLO}$ appears to be the one-half of the Fröliher-Nijenhuis bracket of $A \otimes \zeta$ [2]. In fact, the computation shows

$$
[A \otimes \zeta, A \otimes \zeta]=\left[u\left(p_{\xi}-\varepsilon u_{z}\right)-p\left(u_{\xi}-\varepsilon u_{z}\right)\right] \mathrm{d} x \wedge \mathrm{d} y \otimes \zeta=\mathbf{R} \mathrm{d} x \wedge \mathrm{d} y \otimes \zeta .
$$

\section{Conclusion}

Resuming, we can say that in case of photon-like objects Frobenius integrability viewpoint suggests to make use of one completely integrable three-dimensional distribution (respectively Pfaff system) consisting of one isotropic and two spacelike vector fields (respectively one-forms), such that the corresponding two-dimensional spatial subdistribution $\left(\bar{A}, \bar{A}^{*}\right)$ (respectively Pfaff system $\left(A, A^{*}\right)$ ) defines a completely integrable system, and the rest two two-dimensional subdistributions $(\bar{A}, \bar{\zeta})$ and $\left(\bar{A}^{*}, \bar{\zeta}\right)$ (respectively Pfaff systems $(A, \zeta)$ and $\left.\left(A^{*}, \zeta\right)\right)$ are NONintegrable in general and give the same (up to a sign) curvature. This curvature may be used to build quantities, physically interpreted as energy-momentum internal exchanges between the corresponding two subsystems $(\bar{A}, \bar{\zeta})$ and $\left(\bar{A}^{*}, \bar{\zeta}\right)$ (respectively $(A, \zeta)$ and $\left.\left(A^{*}, \zeta\right)\right)$. Moreover, rotational component of propagation, i.e., spin properties, will be available only if the curvature $\mathbf{R}$ is nonzero, i.e., only if an internal energy-momentum exchange takes place.

\section{References}

[1] Kushner A. Lychagin V. and Rubtsov V., Contact Geometry and Non-linear Differential Equations, Cambriddge Univ. Press, Cambridge 2007.

[2] Michor P., Topics in Differential Geometry, Graduate Studies in Mathematics vol. 93, AMS, Providence, 2008.

[3] Donev S. and Tashkova M., From Maxwell Stresses to Photon-like Objects through Frobenius Curvature Geometrisation of Local Physical Interaction, arXiv e-print:math-ph/0902.3924; see also: math-ph: 0806.4058, 0205046; hep-th: 0705.4170, 0508091, 0403244; physics: 0604021. 
[4] Donev S. and Tashkova M., Complex Structures in Electrodynamics, J. Geom. Symm. Phys. 7 (2006) 13-36.

Stoil Donev

Institute for Nuclear Research

and Nuclear Energy

Bulg. Acad. Sci.

Blvd. Tzarigradsko chaussee 72

1784 Sofia, Bulgaria

E-mail address: sdonev@inrne.bas.bg

Maria Tashkova

Institute for Nuclear Research

and Nuclear Energy

Bulg. Acad. Sci.

Blvd. Tzarigradsko chaussee 72

1784 Sofia, Bulgaria

E-mail address: mtashkova@inrne.bas.bg 